\title{
Einführung in die Neuregelungen für Zulassung und Gebrauch von Pestiziden
}

\author{
Henning Friege
}

Erhalten: 14. Oktober 2009/Akzeptiert: 19. Oktober 2009/Online veröffentlicht: 18. November 2009

(C) Springer-Verlag 2009

Zusammenfassung In den folgenden Aufsätzen wird die Reform des EU-Rechts im Zusammenhang mit der Zulassung und Anwendung von Pflanzenschutzmitteln diskutiert. Unterschiedliche Standpunkte zu einem Fragenkatalog im Zusammenhang mit dem Reformvorhaben werden jeweils aus Sicht des Umweltbundesamts, der Hersteller von Pflanzenschutzmitteln und einer Umweltorganisation wiedergegeben.

Schlüsselwörter EU-Recht · Gewässer · Pestizide · Pflanzenschutz $\cdot$ Stoffzulassung

\section{Revision of the European regulations on the admission and use of pesticides}

\begin{abstract}
In the following articles, the revision of the European regulations on the admission and use of pesticides is reported. Authors from the German environmental protection agency, the producers of chemicals for crop protection, and of a non-governmental organisation clarify their views on the revision with respect to a number of questions.
\end{abstract}

Keywords Admission of chemicals · Crop protection · European regulation $\cdot$ Pesticides $\cdot$ Water

Verantwortlicher Herausgeber: Henning Friege

Im Rahmen der Beitragsserie „Regulatorische Ökotoxikologie“, Hrsg. Tobias Frische, Jan Ahlers, Bettina Hitzfeld

H. Friege $(\bowtie)$

Stadtwerke Düsseldorf AG,

Höherweg 100, 40233 Düsseldorf, Deutschland

E-Mail: hfriege@swd-ag.de

\section{Präambel EU-Pestizidpaket formal vom Ministerrat angenommen}

Am 24. und 25. September 2009 hat der EU-Ministerrat das sogenannte EU-Pestizidpaket formal angenommen. Das Europäische Parlament hatte das Paket bereits am 13. Januar 2009 in zweiter Lesung verabschiedet.

Das Paket besteht aus einer Verordnung über das Inverkehrbringen von Pflanzenschutzmitteln und einer Richtlinie zum nachhaltigen Einsatz von Pestiziden.

Die getroffene Regelung zu der Verschärfung der Zulassungskriterien für Pflanzenschutzmittel und deren Anwendung sind ein wichtiger Schritt in Richtung einer Harmonisierung des Pflanzenschutzrechts mit dem Wasserrecht.

Für die kommunalen Wasserversorgungsunternehmen haben die Regelungen hohe Relevanz. Die Sicherung der Qualität der Rohwasserressourcen ist von grundlegender Bedeutung, um Trinkwasser möglichst naturnah und ohne kostenintensive Aufbereitung als Lebensmittel zur Verfügung zu stellen.

$\mathrm{Zu}$ den Umsetzungsfristen des Pestizid-Paketes:

Die Verordnung über das Inverkehrbringen von Pflanzenschutzmitteln tritt am zwanzigsten Tag nach ihrer Veröffentlichung im EU-Amtsblatt in Kraft, gilt jedoch erst 18 Monate nach dem Inkrafttreten. Dann ist sie in allen ihren Teilen verbindlich und gilt in jedem Mitgliedsstaat. Zum selben Zeitpunkt wird die Richtlinie 91/414/EWG vom 15. Juli 1991 über das Inverkehrbringen von PSM aufgehoben.

Die Richtlinie zum nachhaltigen Einsatz von Pestiziden tritt am Tag nach der Veröffentlichung im EU Amtsblatt in Kraft und muss bis spätestens zwei Jahre nach Inkrafttreten durch nationale Rechts- und Verwaltungsvorschriften umgesetzt werden. 


\section{Aktuelle EU-Reform}

Während Hersteller und gewerbliche Verbraucher wie auch Behörden sich auf die neuen EU-weiten Regelungen nach Verabschiedung von REACH einstellen, hat das Europäische Parlament soeben eine Reform der Regelungen für Zulassung und Gebrauch von Pestiziden (Pflanzenschutzmittel) verabschiedet. Daneben läuft auch eine Diskussion über die Regelungen für sonstige Biozide, z. B. Holzschutzmittel. Pestizide unterliegen als Stoffe, die bestimmungsgemäß in die Umwelt eingebracht werden, seit jeher einer spezifischen Gesetzgebung.

Die EU-Kommission hat mit der Ausgestaltung der neuen bzw. überarbeiteten Pestizidgesetze auch eine politische Strategie, die „Thematische Strategie zur nachhaltigen Nutzung von Pestiziden“ erarbeitet (EU-Kommission 2006).

Welche Probleme waren aus Sicht der EU vordringlich zu lösen?

- Nach wie vor gibt es Besorgnisse in der Öffentlichkeit bei Pestizidfunden in Lebensmitteln. Es ist zwar klar, dass eine intensive, auf die Produktion möglichst preiswerter Nahrungsmittel ausgerichtete Landwirtschaft nicht ohne Pestizide auskommt - diese Folgen der Nachfrage nach billigen Nahrungsmitteln wollen die Verbraucher aber trotz oft sinkender Grenzwerte für Pestizidrückstände in Lebensmitteln nicht tragen. Deshalb fördert die EU alternative Methoden der Landbewirtschaftung, die mit weniger Pestizideinsatz auskommen. Außerdem schlug die Kommission die Substitution von kritischen durch weniger gefährliche Mittel vor.

- Nach wie vor gibt es Berichte über die Fehlanwendung von Pestiziden, z. B. zu nahe an Gewässern, zu falschen Zeitpunkten wie auch über den Vertrieb nicht mehr zugelassener Mittel (UBA 2006). Diese in Deutschland gut dokumentierten Probleme (Lahl 2006) sind auf andere EU-Länder vermutlich übertragbar. Ob die kaum sinkende Belastung von Grundwässern mit Pflanzenschutzmittelwirkstoffen ebenfalls auf Fehlanwendungen zurückgeht oder eine Auswirkung der Anwendung bei „guter fachlicher Praxis“ ist, sei dahin gestellt. Die Kommission zielte daher u. a. auf verstärkte Kontrollen beim Vertrieb und bei der Verwendung von Pestiziden wie auch auf Anwenderschulungen und das Verbot bestimmter Ausbringungstechniken.

Die Kommission stellte fest, dass sich ihre neuen Ziele nur teilweise durch eine Reform der bestehenden Regelungen zum Inverkehrbringen von Pflanzenschutzmitteln (Zulassungsrichtlinie) realisieren lassen. Sie schlug daher eine neue Rahmenrichtlinie für die Anwendung von Pestiziden vor. Im Gegensatz zu der von den nationalen Gesetzgebern in entsprechende Regelungen umzusetzenden Rahmenrichtlinie werden die novellierten Zulassungsregelungen zukünf- tig als Verordnung nach Verabschiedung direkt vollziehbares europäisches Recht.

Diese Harmonisierung hat Vor- wie Nachteile: Länderspezifische Regelungen gibt es nicht mehr - das ist bei den unterschiedlichen biologischen und geochemischen Gegebenheiten in Europa problematisch. Andererseits ist die Gefahr von Wettbewerbsverzerrungen deutlich geringer.

Die EU-Agrarminister einigten sich am 23. Juni 2008 über die Verordnung zum Inverkehrbringen von Pflanzenschutzmitteln und verabschiedeten schließlich am 15. September 2008 einen gemeinsamen Standpunkt.

Am 13. Januar 2009 hat das Europaparlament nunmehr in zweiter Lesung das komplette Paket verabschiedet (Europäisches Parlament 2009) und der Ministerrat dürfte dem in Kürze zustimmen. Das „Paket“ umfasst die Verordnung über das Inverkehrbringen von Pflanzenschutzmitteln sowie die Richtlinie zum nachhaltigen Einsatz von Pestiziden. Diese Regelungen haben für Landwirtschaft, Hersteller und Verbraucher, aber z. B. auch für die Wasserversorger hohe Relevanz. Alle Wirkstoffe von Pflanzenschutzmitteln, die potenziell als krebserregend, erbgut- oder fortpflanzungsschädigend eingestuft sind, werden grundsätzlich verboten. Auch hormonell wirksame Substanzen (endokrine Disruptoren) stehen auf der schwarzen Liste. Für Wirkstoffe, die die Entwicklung von Immun- oder Nervensystem schädigen, ist eine strenge Sicherheitsprüfung vorgesehen. Entsprechend klassifizierte Wirkstoffe unterliegen erstmals einem Substitutionsgebot. Einige negative umweltrelevante Stoffeigenschaften werden ebenfalls für einen Ausschluss oder eine Substitution herangezogen, z. B. hohe Persistenz und Bioakkumulierbarkeit.

Um die unterschiedlichen geochemischen und biologischen Gegebenheiten zu berücksichtigen, wird Europa im Hinblick auf die Pestizidzulassung in drei geografische Zonen eingeteilt. Es ist vorgesehen, dass die Mitgliedstaaten in einer Zone obligatorisch eine gegenseitige Anerkennung von Zulassungen aussprechen. Allerdings können Mitgliedstaaten beantragen, in ihrem Hoheitsgebiet unter bestimmten Bedingungen ein ansonsten zugelassenes Pestizid zu verbieten.

Die Richtlinie zum nachhaltigen Einsatz soll den Rahmen für eine harmonisierte Anwendung von Pestiziden setzen, dessen zentrales Ziel die Risikoreduktion darstellt. Beispielsweise sollen die Mitgliedsstaaten Verbote oder Anwendungsbeschränkungen in spezifischen Gebieten zum Schutz von Gewässern, Biodiversität und an Gewässern aussprechen, den Pflanzenschutz in der Landwirtschaft zukünftig nach integrierten Managementverfahren durchführen und oder die Ausbringung von Pestiziden mit Flugzeugen untersagen.

\section{Die Sicht der Beteiligten}

Das „Paket“ wurde im Parlament mit einer überwältigenden Mehrheit verabschiedet. Seine Befürworter sprechen von 
der weltweit strengsten Pestizidgesetzgebung. Wie wird sich das neue Recht auswirken?

Wesentliche Akteure sind die Hersteller bzw. Importeure, die Behörden, die Landwirtschaft und die in diesem Bereich aktiven Umweltverbände.

In den folgenden Beiträgen sollen Antworten aus der Sicht der Akteure auf folgende Fragestellungen gegeben werden:

1. Wie werden die Auswirkungen der bisherigen nationalen Reduktionsprogramme für die Ausbringung von Pestiziden eingeschätzt, und wie wird sich dies mit der neuen Rechtslage ändern? Wie können sinnvolle Reduktionsprogramme aussehen?

2. Welche Folgen würde die Einführung eines Substitutionsgebots und der vergleichenden Bewertung von Wirkstoffen haben? Welche Erfahrungen aus der Vergangenheit sprechen für diese geplante Verschärfung der EU-Regelungen?

3. Welche Probleme erwarten Hersteller und Importeure mit dem neuen Pestizidrecht?

4. Wie hat sich die Rückstandssituation für Nahrungsmittel und Umweltmedien in den vergangenen zehn Jahren entwickelt? Welche Trends lassen sich erkennen und auf die bisherige Ausbringungspraxis zurückführen? Wie können sich die neuen Anwendungsregelungen, die Anwenderausbildung etc. auf die Rückstandssituation von Nahrungsmitteln, vor allem aber auf Grund- und Oberflächenwasser auswirken? Welche Vorteile werden die neuen Regelungen für Verbraucher und Umwelt bringen? Welche Nachteile werden für die Landwirtschaft gesehen?

Für die folgenden Stellungnahmen konnten folgende Expertinnen und Experten gewonnen werden:

- Frau Dr. Dietlinde Großmann, Abteilung Pflanzenschutzmittel des Umweltbundesamts, Dessau (http://www. uba.de),

- Dr. Herbert Resseler, Dr. Otto Klein und Dr. Manfred Lefèvre für den Industrieverband Agrar (http://www.iva.de), dort jeweils Sprecher der Arbeitskreise Ökochemie bzw. Rückstandsfragen bzw. Technik,

- Frau Susanne Smolka, Referentin bei PAN Germany. Das Pesticide Action Network ist eine seit 25 Jahren arbeitende Organisation mit weltweiter Vernetzung (http://www. pan-germany.org).

\section{Literatur}

EU-Kommission (2006) Entwurf zur Thematischen Strategie zur nachhaltigen Nutzung von Pestiziden. $\mathrm{KOM}(2006) 372$, Brüssel

Europ. Parlament (2009) www.europarl.de/presse/pressemitteilungen/ quartal2009 1/PM 090113 1a

Lahl U (2006) Anwendungen von Pflanzenschutzmitteln in der Landwirtschaft. Umweltwiss Schadst Forsch 18(1):41-48

UBA (Umweltbundesamt) (2006) Anwendung von Pflanzenschutzmitteln in der Landwirtschaft, Pressemitteilung vom 10. Januar 2006. http://www.bvl.bund.de/cln_007/nn_492018/DE/04_Pflanzenschutzmittel/05_KontrolleUeberwachung/01_PSM_Kontrollprg/psm_KontrollPrg_node.html_nnn=true 